\title{
Effects of HMGB1 on proliferation and apoptosis of human brain glioma CD133 cells
}

\author{
Zhao WP ${ }^{1,2}$, Chen $\mathrm{QX}^{2}$ \\ Neurosurgery Department, Inner Mongolia People’s Hospital, Huhhot, Inner Mongolia. \\ chenqianxue2013@163.com
}

\begin{abstract}
OBJECTIVE: To understand the effects of HMGB1 recombinant lentivirus vector on proliferation and apoptosis on CD133 cells after transfecting a human glioma cell strain CD133.

METHOD: The CD133 human glioma cell strain cultured in vitro was transfected with HMGB1 lentiviral vector and empty vector, respectively, while CD133 cells cultured normally were used as the blank control. Changes in HMGB1 protein level were detected with western blot hybridization; apoptosis was detected with a flow cytometer; the cell proliferation was analysed with MTT method.

RESULTS: The results from the western blot hybridization indicated that the expression level of HMGB1 protein in the group infected with HMGB1 recombinant over-expressed lentiviral vector increased significantly $(p<0.05)$ compared with the blank control and CD133 cells transfected with the empty vector. The absorbance value of cell proliferation in the group infected with HMGB1 over-expressed lentiviral vector increased gradually on days $1,2,3,4$ and 5, but the difference was significant on day 2 after infection and later compared with the blank control group and negative control group $(p<0.05)$. Based on detection with a flow cytometer, the apoptosis rate of CD133 cells transfected with HMGB1 over-expressed viral vector was significantly higher than that in the blank control group and negative control group $(p<0.05)$.

CONCLUSION: The lentiviral expression vector has very high transfection efficiency in human glioma cell line CD133 and the infection is able to significantly up-regulate the expression and activation of HMGB1. Over-expression of HMGB1 is able to inhibit proliferation of tumor cells and promote apoptosis (Tab. 2, Fig. 6, Ref. 30). Text in PDF www.elis.sk. KEY WORDS: HMGB1, CD133, glioma, cell proliferation, apoptosis.
\end{abstract}

\section{Introduction}

Brain glioma is the most common primary tumor among central nervous system tumors in adults. It has a high morbidity and accounts for approximately $40-50 \%$ of intracranial tumors. More than $3 / 4$ of cases are malignant (1). Surgical excision is the most effective treatment means for glioma. However, the glioma is not able to be completely removed by surgery and it is susceptible to relapse, short survival time, high mortality, and poor prognosis even though it can be completely removed under naked-eye examination due to the fact that glioma is characterized by diffuse infiltrative growth, no apparent boundary with normal brain tissue, frequent invasion of nervous structure, etc. (2-3). However, chemotherapy and radiotherapy are not able to kill the cancer cells with high degree specificity and may give rise to severe adverse reactions (4). Thus, the existing surgery, radiotherapy, chemotherapy or other comprehensive therapies are not able to treat glioma radically. The brain glioma is still a refractory disease in the field of neurosurgery and the explanation of its pathogenesis

${ }^{1}$ Neurosurgery Department, Inner Mongolia People's Hospital, Huhhot, Inner Mongolia, and ${ }^{2}$ Neurosurgery Department, Renmin Hospital of Wuhan University, Wuhan, Hubei Province

Address for correspondence: Q.X. Chen, Neurosurgery Department, Renmin Hospital of Wuhan University, Zhangzhidong Road No. 99, Wuhan, Hubei Province, 430060, Mongolia. and exploration of new treatment means have become two research highlights in neurosurgery.

In recent years, with the development of disciplines such as molecular biology, molecular genetics etc., a number of genes closely associated with occurrence, progression and prognoses of tumors have been found. Hence, people have attempted to improve the curative effect in treatment of glioma by gene therapy in exploring the treatment of malignant tumors. The gene therapy is a biomedical technique where normal human genes or therapeutic human genes are transferred into human target cells by certain means to correct the gene defects or exert the therapeutical effect for treatment of diseases (5-6). Thus, selecting an effective target gene is the most important factor for achieving the desired therapeutic effect.

To further study the role of HMGB1 in the occurrence and progression of glioma and the feasibility of serving as a genetic therapeutic target of human brain glioma, the paper studies the role of HMGB1 gene in biological characteristics of glioma and provides experimental bases for whether it can serve as a new target in the treatment of glioma.

\section{Material and methods}

Material

The malignant gliocytoma in vitro cell line CD133 was purchased from Shanghai Xitang Biotechnology Co., Ltd.; HMGB 
gene plasmid was purchased from Bei Jing Ruizekang Biotechnology Co., Ltd.; TaqDNA polymerase and dNTP were purchased from Shanghai Meiji Biotechnology Co., Ltd.; the DL2000 DNA Marker was purchased from Shanghai Shrek Biotechnology Co., Ltd.; plasmid extraction kit was purchased from U.S. Sigma Company; fetal bovine serum and DMEM culture medium were purchased from Hangzhou Biowish biotechnology Co., Ltd.; Trizol reagent was purchased from Beijing Bole Bioscience Development Co., Ltd.; the imager was purchased from LEAD-TECH (Shanghai) Scientific Instrument Co., Ltd.; high speed freezing centrifuge was purchased from Beijing Jingli Centrifuge Co., Ltd.; horizontal electrophoresis apparatus was purchased from Shanghai Aoxi Technology Instrument Co., Ltd.; RealPlex4 real-time fluorescence quantification PCR amplifier was purchased from U.S. Becton Dickinson Company; Multiskan MK3 fully-automatic microplate reader was purchased from U.S. Hach Company; flow cytometer was purchased from U.S. MEDICA Company.

\section{Construction of HMGB1 over-expressed lentiviral vector}

The expression vector was incised by EcoR I and Nhe 1. The EGFP fragment was incised. The vector fragments of approximately $12.4 \mathrm{~kb}$ were recovered after the enzyme-incision product had been electrophoresed. Based on the reference sequence of the target gene mRNA, the intron-flanking primer was designed with the primer premier 5.0 software (7) and the specific sequence was:

Forward: 5'-CCGCCCCCAGGGATTCGCAGGCGTAGTAGGGGGGA-3'

Reverse: 5'-GATTGCTTCGTAGTACCGGTAGCATCAGCACTTCT-3'

A volume of $0.5 \mu \mathrm{l}$ of $10 \mathrm{mM}$ dNTP mixture, $10 \mu \mathrm{l}$ of $10 \times \mathrm{PCR}$ buffer, $1 \mu \mathrm{l}$ of Taq DNApolymerase, $1 \mu \mathrm{l}$ of HMGB1 plasmid solution, $32.5 \mu \mathrm{l}$ of $\mathrm{ddH}_{2} 0,1 \mu \mathrm{l}$ of upstream primer, and $1 \mu \mathrm{l}$ of downstream primer were added to the PCR reaction system. The solution was well mixed and placed into the reaction holes of the PCR amplifier. The reaction conditions were as follows: initiallydenatured $95^{\circ} \mathrm{C} \times 3 \mathrm{~min}$, denatured $94^{\circ} \mathrm{C} \times 1 \mathrm{~min}$, annealed $59^{\circ} \mathrm{C} \times 1$ min, extended $72{ }^{\circ} \mathrm{C} \times 1 \mathrm{~min}, 30$ cycles. Electrophoresis identification was performed for the PCR product with DL2000 marker as reference. The HMGB1 gene fragments were recovered and joined in the expression vector, incubated at $37^{\circ} \mathrm{C}$ for $15 \mathrm{~min}$, and then incubated at $50{ }^{\circ} \mathrm{C}$ for $15 \mathrm{~min}$. An appropriate amount of transformed bacteria solution was taken and uniformly applied onto the LB agar plate, stored at room temperature until the liquid was absorbed, and then incubated in an incubator at constant temperature of $37^{\circ} \mathrm{C}$ for 24 hours.

\section{Identification of positive clone}

The primers designed and synthesized were as follows: (8):

Primer (+)5'-GTGAGGGTCAAATGCAATT-3';

Primer (-)5'-AGCAACACAGCGTAAAAGG-3'.

The transformants growing on the plate were selected and re-suspended in $10 \mu \mathrm{LB}$ culture solution and $1 \mu \mathrm{l}$ was taken as a template for PCR identification in the bacterial colony. The reaction system was as follows: $10 \mathrm{mM} \mathrm{dNTP} \mathrm{mixture} 1.6 \mu \mathrm{l}, 10 \times \mathrm{PCR}$ buffer $2 \mu 1$, Taq DNApolymerase $0.1 \mu$, Primer (+) and Primer (-)
$0.8 \mu \mathrm{l}$ each, bacteria solution supernatant $1 \mu \mathrm{l}, \mathrm{ddH}_{2} 013.7 \mu \mathrm{l}$. The solution placed into the reaction holes of the PCR amplifier. The reaction conditions were as follows: initially-denatured $9{ }^{\circ} \mathrm{C} \times 3$ min, denatured $94{ }^{\circ} \mathrm{C} \times 1 \mathrm{~min}$, annealed $5{ }^{\circ} \mathrm{C} \times 1 \mathrm{~min}$, extended 72 ${ }^{\circ} \mathrm{C} \times 1 \mathrm{~min}, 30$ cycles. The PCR products were subject to agarose gel electrophoresis.

\section{Infecting CD133 cells with lentivirus}

The CD133 cells were resuscitated. The old culture medium was discarded. A volume of $5 \mathrm{ml}$ PBS solution was added. The cell growth face was washed. The PBS solution was discarded. A volume of $2 \mathrm{ml}$ pancreatin digestive fluid was added for 5 min digestion. The digestive fluid was pipetted and the complete medium containing $10 \%$ fetal calf serum and double-antibody was added.

The cells on the flask wall were pipetted repeatedly and gently to make uniform cell suspension. The cell suspension was placed in a culture flask as per $1: 3$ for passaging and amplified for 3-5 passages.

The passaged CD133 cells were inoculated into 16-well culture plate with $2 \times 10^{5}$ in each well, cultured in an incubator containing $5 \% \mathrm{CO}_{2}$ for $12 \mathrm{~h}$ at $37^{\circ} \mathrm{C}$. An appropriate amount of lentivirus, and $5 \mu \mathrm{g} / \mathrm{ml}$ polybrene (infection enhancer) were added when the cell fusion reached $80 \%$ and the cell state was satisfactory. The culture medium was replaced after $48 \mathrm{~h}$. The expression of the reporter gene GFP on the lentivirus was observed (9).

The CD133 cells were divided into three experimental groups as follows: 1) blank control group: group of CD133 cells not infected with virus; 2) negative control group: group CD133 cells not transfected with empty viral vector; 3 ) experimental group: CD133 cells not infected with HMGB1 recombinant lentivirus vectors.

\section{Detecting the changes in the HMGB1 protein before and after} transfection with Western blot hybridization

The cells cultured before and after infection in various groups were collected, placed on the ice for sufficient pyrolysis after addition of $1 \mathrm{~m} 1$ cellular total protein extraction buffer, and transferred into a $1.5 \mathrm{ml} \mathrm{EP}$ tube. It was centrifuged in a refrigerated high-speed centrifuge at $12000 \mathrm{rpm}$ for $10 \mathrm{~min}$. The supernatant was taken and sample buffer was added for later use. The protein was transferred in a transfer tank and stained inversely. Immunodetection was performed with an anti-body conjugate (GAPDH as internal control). A standard curve was plotted on the basis of the distance between standard electrophoresis band of different protein molecular weights and the sample points. The molecular weight of the protein to be detected was determined by contrasting the distance from the hybridization band to the sample hole and that mentioned above. The peak area value of each hybridization image was detected with an optical density scanner. The ratio of the protein to be tested to the peak area value of GAPDH was used to represent the expression level of the protein to be tested.

\section{Detecting cell proliferation with MTT method (thiazolyl blue col- orimetric analysis)}

The cells in logarithmic growth metaphase with a good growth state of the three groups: the uninfected group, empty vector in- 


\section{0-485}

fection group and the HMGB1 recombinant virus vector infection group were harvested, made into single-cell suspension using cell culture fluid, and inoculated into a cell culture plate with $1 \times 10^{5}$ cells per well. Eight parallel wells were arranged for each group. They were cultured in an incubator containing $5 \% \mathrm{CO}_{2}$ at $37^{\circ} \mathrm{C}$. Cell growth was detected with MTT method on days 1 to 5. One culture plate was taken out each day. A volume of $20 \mu \mathrm{l}$ of freshly-prepared MTT solution was added to each well, mixed well, and cultured for 4 hours continuously. Then the culture was terminated. The supernatant in the hole was pipetted, $100 \mu \mathrm{l}$ of dimethyl sulfoxide was added to each well and vibrated for 10 min. The optical density was detected on ELISA detector after the crystal substance had been dissolved completely. The OD value expressed the degree of cell proliferation (10).

\section{Analysing the apoptosis rate with a flow cytometer}

The cell culture fluid was pipetted into a proper centrifuge tube and shaken gently after addition of PBS with a pipette. The adherent cells were washed once, and PBS buffer was discarded. The cells were digested with pancreatin cell digestion solution, pipetted up and down gently after addition of cell culture solution, transferred into a centrifuge tube with a pipette after the cells had been suspended completely, and centrifuged at $1000 \mathrm{rpm}$ for $10 \mathrm{~min}$. The supernatant was discarded. The cells were collected, re-suspended gently with PBS, and counted. 50,000-100,000 cells were taken and centrifuged at $1000 \mathrm{rpm}$ for $10 \mathrm{~min}$. The supernatant was discarded. A volume of $5 \mu$ l of Annexin V-FITC binding buffer was added to gently re-suspend the cells. The cells were incubated in the dark for 10 minutes. They were centrifuged at 1,000 rpm for $10 \mathrm{~min}$. The supernatant was discarded. A volume of $190 \mu$ l of Annexin V-FITC binding solution was added to gently re-suspend the cells. A volume of $10 \mu \mathrm{l}$ of propidium iodide (PI) staining solution was added, well mixed gently, and placed in an ice-bath in the dark. Then it was detected with flow cytometer. Annexin V-FITC was green fluorescence and PI was red fluores-

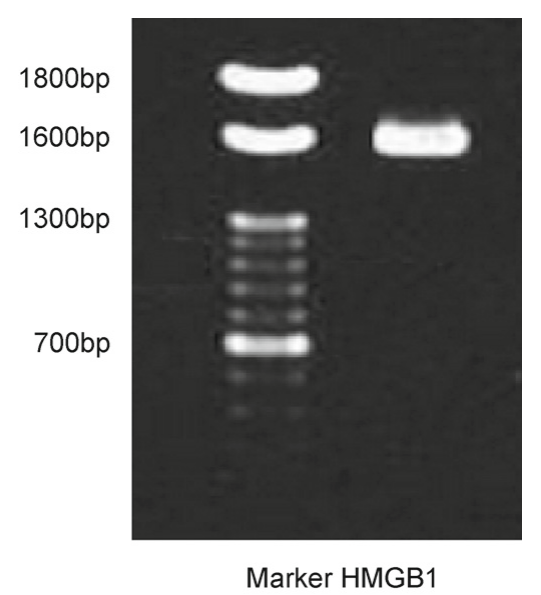

Fig. 1. Electrophoretogram for PCR product of HMGB1. An obvious HMGB1 band was present at 1,600 bp as reported in literature, which demonstrated that the MGB1 over-expressed lentiviral vector was constructed successfully.

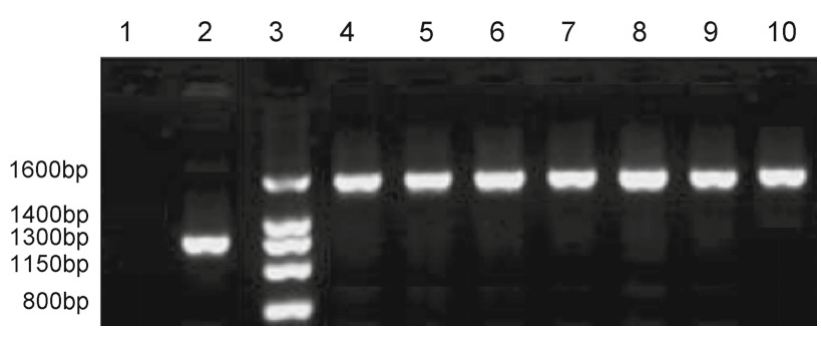

Fig. 2. PCR identification of HMGB1 recombinant positive clones. 1) negative control ( $\left.\mathrm{ddH}_{2} \mathrm{0}\right)$; 2 ) negative control (empty vector group); 3) DL2000 DNA Marker; 4-10. 7 transformants selected. The positive clonal PCR fragment of the HMGB1 fragment appeared at 1,622 bp, which was basically the same as the position of the pure HMGB1 fragment; the size of the negative clonal PCR fragment not joined with the HMGB1 fragment was 1,131 bp, which indicated that the HMGB1 over-expressed lentiviral recombinant positive cloning was successful.

cence. The cells were centrifuged at $1,000 \mathrm{rpm}$ for $10 \mathrm{~min}$ when detected under a fluorescence microscope. The cells were collected, re-suspended gently with $100 \mu \mathrm{l}$ Annexin V-FITC binding solution, smeared, and observed under the fluorescence microscope.

\section{Statistical analysis}

The statistical results were expressed with mean \pm standard deviation $( \pm \mathrm{s})$. The SPSS 15.0 statistical software was used for statistical analysis. Based on the different methods for data collection and different requirements of statistical treatment, oneway analysis of variance (one-way ANOVA test), F test, SNK-q test etc. were selected. $\mathrm{p}<0.05$ was considered statistically significant.

\section{Results}

Identification of construction of over-expressed lentiviral vector in HMGB1

The size of the PCR product of HMGB1 was 1,524 bp. Agarose gel electrophoresis was performed. It was observed in the gel imaging system where the target band was present at approximately $1,600 \mathrm{bp}$ consistent with the size of the open reading frame of HMGB1 (Fig. 1).

\section{Identification of recombinant positive clone}

The recovered target gene fragments were joined together with the pLV-GFP vector incised by EcoR 1 and Nhe I enzymes. The joining product was used to convert the DH5a Escherichia coli. The recombinant positive clones were selected and subjected to PCR identification. The recombinant bacterial clonal PCR products were subjected to agarose gel electrophoresis while one clear band in Track 4-9 and Track 11 was observed. The electrophoretic mobility of the PCR product band was slower than the PCR product band in Track 2, i.e. the empty vector group, which indicated that the 4-11 tracks were positive colonies of fragments. The size of positive clonal PCR fragment joined with the HMGB1 fragment was $1,622 \mathrm{bp}$; the size of negative clonal PCR fragment not joined to the HMGB1 was 1,131 bp (Fig. 2). 
Tab. 1. Expression of the HMGB1 protein before and after the CD133 cells were infected with lentivirus.

\begin{tabular}{lccc}
\hline & $\begin{array}{c}\text { Blank Control } \\
\text { Group }\end{array}$ & $\begin{array}{c}\text { Negative Control } \\
\text { Group }\end{array}$ & $\begin{array}{c}\text { HMGB1-CD133 } \\
\text { Infection Group }\end{array}$ \\
\hline $\begin{array}{l}\text { Expression Level } \\
\text { of HMGB1 }\end{array}$ & $0.192 \pm 0.031$ & $0.252 \pm 0.043$ & $0.694 \pm 0.061^{*}$ \\
\begin{tabular}{l} 
Protein \\
\hline Compared with the blank control group and the negative control group, ${ }^{*} \mathrm{p}<0.05$
\end{tabular}
\end{tabular}

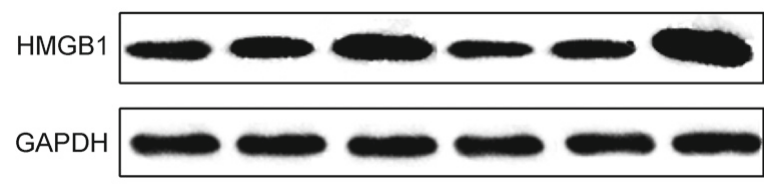

Fig. 3. HMGB1 hybridization result of CD133 Cells and cells respectively transfected with the empty vector and HMGB1 over-expressed viral vector. 1) blank control group untransfected; 2) negative control group; 3) infection group; 4-6) duplications.

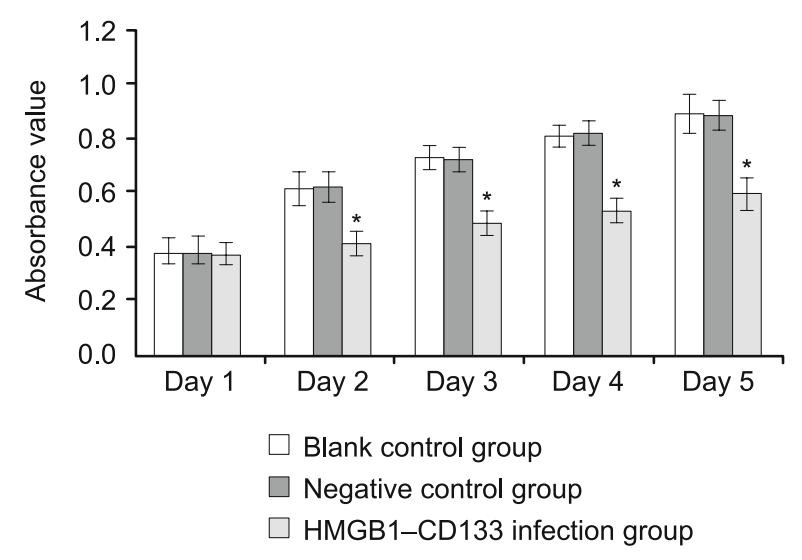

Fig. 4. Effect of HMGB1 over-expressed lentiviral vector on proliferation of CD133 Cells. Compared with the blank control group and negative control group, $* \mathbf{p}<0.05$.

Detecting the changes in the HMGB1 protein before and after transfection with Western blot hybridization

For the blank control and CD133 cells respectively transfected with the empty vector and HMGB1 over-expressed lentiviral vector, the Western blot hybridization result indicated that the level of the HMGB1 protein in the group infected with HMGB1 recombinant over-expressed lentiviral vector increased significantly $(\mathrm{p}<$ 0.05 ) compared to the blank control and CD133 cells transfected with the empty vector, which demonstrated that the pLV-HMGB1 recombinant over-expressed lentiviral vector was able to effectively infect the CD133 cells and the exogenous HMGB1 could be correctly expressed in the CD133 cells. It demonstrated that the pLV-HMGB1 over-expressed lentiviral vector had high infection efficiency in CD133 cells (Fig. 3 and Tab. 1).

\section{MTT experimental result}

The absorbance value of cell proliferation in the group infected with HMGB1 over-expressed lentiviral vector increased gradually on days 1, 2, 3, 4 and 5, but the difference was significant on day 2 after infection and later compared with the blank control group and negative control group $(\mathrm{p}<0.05)$. There was no significant difference between the blank control and negative control group (p>0.05) (Tab. 2 and Fig. 4).

\section{Result of detecting apoptosis with a flow cytometer}

Based on the detection with the flow cytometer, the apoptosis rates of the three groups of CD133 glioma cells were $6.26 \pm 0.54$, $7.53 \pm 0.81$, and $18.51 \pm 2.33$, respectively. The apoptosis rate of CD133 cells transfected with HMGB1 over-expressed viral vector was significantly higher than that of the blank control group and negative control group $(\mathrm{p}<0.05)$, and the difference was statistically significant. There was no significant difference between the blank control group and negative control group $(p>0.05)$, which indicated that the transfer of exogenous HMGB1 could induce early apoptosis of CD133 cells (Figs 5 and 6).

\section{Discussion}

Similar to the tumors in other sites of human body, the occurrence and progression of malignant brain glioma is a very complex process under combined action of various comprehensive factors, and it concerns the expression abnormality or inactivation of various tumor-related genes (11). Glioma is essentially a disease with multigene abnormality and its occurrence is due to the fact that abnormal activation of protooncogene, high-expression and /or deletion and inactivation of cancer suppressor gene cause abnormalities in cell signaling conduction path, change in cell cycles, extension of life cycle, defect of apoptosis etc. These abnormalities lead to incontrollable cell proliferation and malignant transformation (12-13). With the progress in molecular biological techniques and development of research on tumor pathogenesis, the gene therapy has become a major highlight in the glioma research field. In recent years, research on gene therapy has developed rapidly. It has been increasingly closely combined with techniques such as stem cell treatment, and immunity-directed treatment. Much progress has been achieved and as a result, partial gene therapeutic regimens have entered the clinical test stage. However, the development of gene therapy is substantially restricted due to such urgent problems

Tab. 2. Effect of HMGB1 over-expressed lentiviral vector on proliferation of CD133 cells.

\begin{tabular}{lcccc}
\hline Group & \multicolumn{3}{c}{${\text { Absorbance Value }\left(\mathrm{A}_{570}\right)}$} \\
\cline { 2 - 5 } & Day 1 & Day 2 & Day 3 & Day 4 \\
\hline Blank Control Group & $0.381 \pm 0.012$ & $0.614 \pm 0.012$ & $0.732 \pm 0.018$ & $0.807 \pm 0.021$ \\
Negative Control Group & $0.379 \pm 0.009$ & $0.618 \pm 0.017$ & $0.724 \pm 0.016$ & $0.812 \pm 0.017$ \\
HMGB1-CD133 Infection Group & $0.372 \pm 0.010$ & $0.411 \pm 0.011^{*}$ & $0.493 \pm 0.009^{*}$ & $0.531 \pm 0.015^{*}$ \\
\hline
\end{tabular}

Compared with the blank control group and negative control group, ${ }^{*} \mathrm{p}<0.05$. 

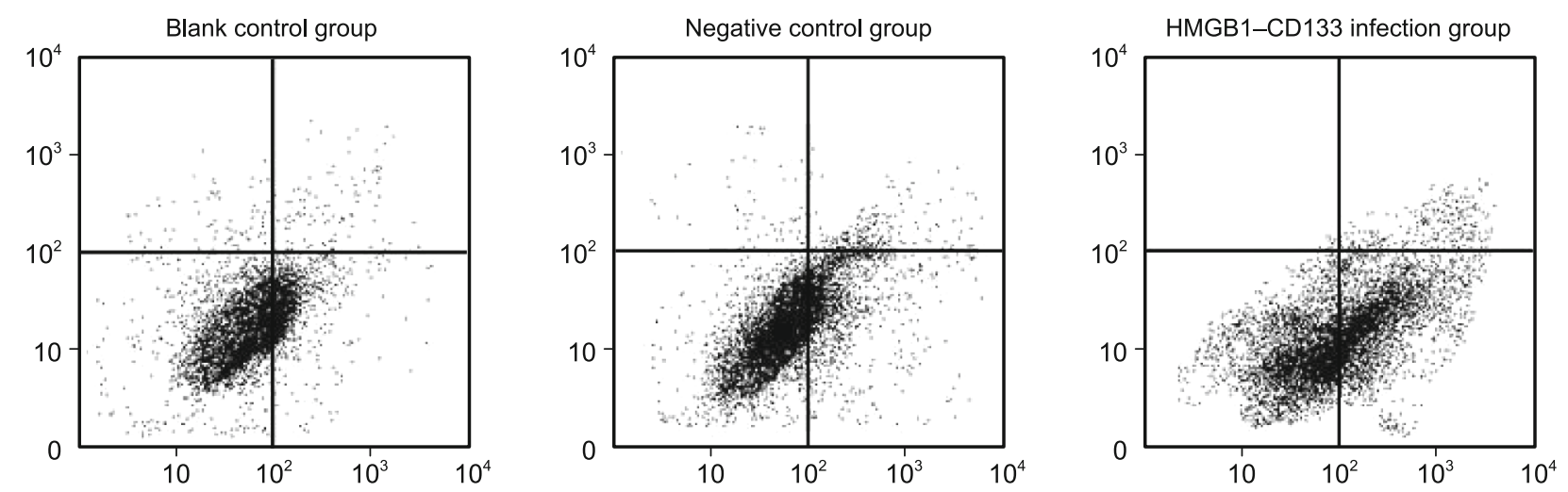

Fig. 5. Detecting Apoptosis with a Flow Cytometer.

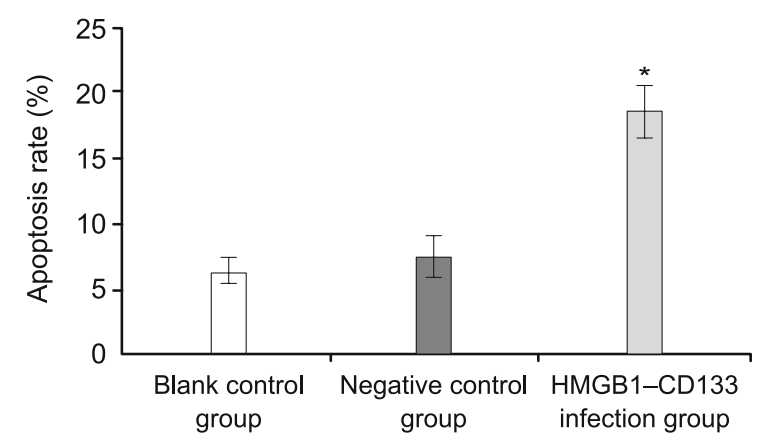

Fig. 6. Quantitative analysis of changes in apoptosis levels before and after transfection of CD133 cells with flow cytometer. Compared with the blank control group and negative control group, ${ }^{*} p<0.05$.

as low vector safety and transfection efficiency, expression time and immunogenicity of exogenous genes etc. (14).

A vector is a vehicle for inserting target genes and transferring them to the host cells for expression or duplication (15). The common vectors include viral and nonviral vectors. Currently, viral vectors are the major tool for gene therapy research and clinical application due to the fact that they have a high transfection efficiency and can be used in vivo and in vitro gene transductions (16). The lentiviral vector is a reverse transcription viral vector that has aroused extensive attention in recent years. In host cells, the lentivirus is able to synthesize cDNA under the action of its own reverse transcriptase with the virus RNA as the template and then synthesize double-strand DNA with cDNA as template. It can be integrated onto the chromosome of the host cells through the effect of the viral integrase and expressed for a long period of time after cyclization (17). Thus, the lentiviral vectors constructed on the basis of lentiviral genome by substituting therapeutic genes and selective markers for genes necessary for duplication have advantages such as high-capacity of the transferred gene fragments, long-term expression of the target gene integrated into the target cell genome, low risk of inducing host immunoreaction, high safety etc. (18-19). In addition, the most significant characteristic is its capacity to infect the dividing cells and non-dividing cells such as nerve cells, hematopoietic stem cells, muscle fiber cells, stem cells, etc. (20-21). In the research, the lentiviral particles of the vector system are of self-inactivating type and will not generate full-length RNA after being integrated into the target cells. They only have the function of carrying target genes. They will neither infect other cells after infecting the target cells nor generate new viral particles using the host cells. In spite of this, the virus still has a potential biological hazard and care should be taken.

The occurrence of glioma is due to the fact that the activation of protooncogenes and inactivation of cancer suppressor genes cause abnormalities in the cell signal transduction pathway and damage the normal and limited life cycle of cells, thus allowing for malignant proliferation of cells and formation of tumors (2223). To clarify the role of HMGB1 in proliferation and apoptosis of glioma cells, the exogenous HMGB1 was transferred into the glioma cell line CD133 and the expression was up-regulated. The proliferation and apoptosis of CD133 cells before and after transfection were observed using flow cytometer and MTT method.

The cell proliferation process is one where the parent cells were replicated to form daughter cells, which is called a cell cycle. The cell cycle is divided into Stage G1, Stage S, Stage G2, and Stage M (24). Stage G1 refers to the period between mitosis completion and start of DNA duplication, which makes preparation for DNA duplication and protein synthesis. The early stage of Stage G1 is called Stage G0. Stage S is critical in cell cycle, i.e. stage of DNA duplication and synthesis, which can reflect the cell proliferation (25). Stage $\mathrm{S}$ accounts for the highest percentage of cell cycle indicating an active DNA synthesis (26). Generally, Stage $\mathrm{S}$ of tumor cells has a higher percentage than that of normal cells and their proliferation activity is stronger than that of the normal cells (27). In the experiment, it is observed that the HMGB1 is able to inhibit proliferation of tumor cells based on MTT experimental detection of CD133 cells before and after transfection. The growth rate of CD133 cells transfected with HMGB1 over-expressed lentiviral vector was significantly lower than that in the untransfected group and negative control group from the second day after transfection, and the inhibition degree was more significant as time elapsed, which indicated that the HMGB1 lentiviral vector could be integrated into the genome of target cells and maintained for a long period of time thus significantly lowering the growth rate of glioma cells CD133. 
Apoptosis refers to autonomous and sequential death of cells controlled by genes to maintain a stable internal environment (28). The apoptosis is a process strictly controlled by multiple genes and these genes are very conservative in species, e.g. in Bcl-2 family, caspase family etc. (29). The tumor pathogenetic mechanism can be understood from the aspect of apoptosis. A series of oncogenes and protooncogenes are activated and over-expressed, which blocks the apoptosis process of tumor cells and impedes the decrease in cells (30). In the experiment, we detected the changes in the apoptosis process of CD133 cells before and after transfection by using Annexin V-FITC and PI double-staining method. The result has shown that early apoptosis occurred in glioma cells and the percentage of apoptotic cells was higher than that in the untransfected group after up-regulating the expression of HMGB1. We have used the empty vector not joining the target gene as a control and found no significant difference between the control group and untransfected group due to the fact that any transfection method may have toxic effect on cells and may cause cell death. It indicates that HMGB1 is highly expressed under normal conditions and exhibits potential activity of cancer suppressor gene; whereas, the percentage of apoptotic cells significantly rises after the exogenous HMGB1 gene is transferred into the CD133 glioma cells with an originally low expression, which indicates that HMGB1 is on the upstream of the regulatory pathway and its activation or over-expression can promote apoptosis of glioma cells.

\section{Reference}

1. Tamura K, Aoyagi M et al. Expansion of CD133-positive glioma cells in recurrent de novo glioblastomas after radiotherapy and chemotherapy. J Neurosurg 2013; 119 (5): 1145-1155.

2. Wei Y, Jiang Y et al. Activation of PI3K/Akt pathway by CD133-p85 interaction promotes tumorigenic capacity of glioma stem cells. Proc Natl Acad Sci USA 2013; 110 (17): 6829-6834.

3. Lehnus KS, Donovan LK et al. CD133 glycosylation is enhanced by hypoxia in cultured glioma stem cells. Int J Oncol 2013; 42 (3): 1011-1017.

4. Yu SP, Yang XJ et al. Enhanced invasion in vitro and the distribution patterns in vivo of CD133+ glioma stem cells. Chin Med J (Engl) 2011; 124 (17): 2599-2604.

5. Yang B, Wang $\mathbf{Y}$ et al. The ultrastructural difference between CD133positive U251 glioma stem cells and normal U251 glioma cells. Ultrastruct Pathol 2012; 36 (6): 404-408.

6. Hua W, Yao Y et al. The CD133+ tumor stem-like cell-associated antigen may elicit highly intense immune responses against human malignant glioma. J Neurooncol 2011; 105 (2): 149-157.

7. Fang KM, Lin TC et al. Enhanced cell growth and tumorigenicity of rat glioma cells by stable expression of human CD133 through multiple molecular actions. Glia 2013; 61 (9): 1402-1417.

8. Ding BS, James D et al. Prominin 1/CD133 endothelium sustains growth of proneural glioma. PLoS One 2013; 8 (4): 150-154.

9. Cheng RB, Ma RJ et al. PTEN status is related to cell proliferation and self-renewal independent of CD133 phenotype in the glioma-initiating cells. Mol Cell Biochem 2011; 349 (2): 149-157.

10. Campos B, Herold-Mende CC. Insight into the complex regulation of CD133 in glioma. Int J Cancer 2011; 128 (3): 501-510.
11. Qin K, Tian G et al. Anti-glioma response of autologous T cells stimulated by autologous dendritic cells electrofused with CD133+ or CD133glioma cells. J Neuroimmunol 2012; 242 (1): 9-15.

12. He J, Shan $\mathrm{Z}$ et al. Expression of glioma stem cell marker CD133 and O6-methylguanine-DNA methyltransferase is associated with resistance to radiotherapy in gliomas. Oncol Rep 2011; 26 (5): 1305-1313.

13. Short SC, Giampieri S et al. Rad51 inhibition is an effective means of targeting DNA repair in glioma models and CD133+ tumor-derived cells. Neuro Oncol 2011; 13 (5): 487-499.

14. Nakano I, Chiocca EA. Finding drugs against CD133+ glioma subpopulations. J Neurosurg 2011; 114 (3): 648-650.

15. Gopisetty G, Xu J et al. Epigenetic regulation of CD133/PROM1 expression in glioma stem cells by $\mathrm{Sp} 1 / \mathrm{myc}$ and promoter methylation. Oncogene 2013; 32 (26): 3119-3129.

16. Angelastro JM, Lame MW. Overexpression of CD133 promotes drug resistance in C6 glioma cells. Mol Cancer Res 2010; 8 (8): 1105-1115.

17. Wan F, Zhang $S$ et al. The utility and limitations of neurosphere assay, CD133 immunophenotyping and side population assay in glioma stem cell research. Brain Pathol 2010; 20 (5): 877-889.

18. Ariga T. A possible turning point in the hematopoietic stem cell gene therapy for primary immunodeficiency diseases? Lentiviral vectors could take the place of retroviral vectors. Expert Rev Clin Immunol 2013; 9 (11): 1015-1018.

19. Geiling B, Vandal G et al. A modular lentiviral and retroviral construction system to rapidly generate vectors for gene expression and gene knockdown in vitro and in vivo. PLoS One 2013; 8 (10): 279-283.

20. Marumoto T, Tashiro A et al. Development of a novel mouse glioma model using lentiviral vectors. Nat Med 2009; 15 (1): 110-116.

21. Low PT, Lai MI et al. Transgene expression from CpG-reduced lentiviral gene delivery vectors in vitro. Gene 2013; 533 (1): 451-455.

22. Ulasov IV, Nandi S et al. Inhibition of Sonic hedgehog and Notch pathways enhances sensitivity of CD133 (+) glioma stem cells to temozolomide therapy. Mol Med 2011; 17 (1): 103-112.

23. Candolf MYagiz K et al. Release of HMGB1 in response to proapoptotic glioma killing strategies: efficacy and neurotoxicity. Clin Cancer Res 2009; 15 (13): 4401-4414.

24. Clement V, Dutoit V et al. Limits of CD133 as a marker of glioma selfrenewing cells. Int J Cancer 2009; 125 (1): 244-248.

25. Chang CJ, Hsu CC et al. Enhanced radiosensitivity and radiationinduced apoptosis in glioma CD133-positive cells by knockdown of SirT1 expression. Biochem Biophys Res Commun 2009; 380 (2): 236-242.

26. Griguer CE, Oliva CR et al. CD133 is a marker of bioenergetic stress in human glioma. PLoS One 2008; 3 (11): 655-658.

27. Rebetz J, Tian D et al. Glial progenitor-like phenotype in low-grade glioma and enhanced CD133-expression and neuronal lineage differentiation potential in high-grade glioma. PLoS One 2008; 3 (4): 1936-1940.

28. Friedman GK, Langford CP et al. Engineered herpes simplex viruses efficiently infect and kill CD133+ human glioma xenograft cells that express CD111. J Neurooncol 2009; 95 (2): 199-209.

29. Soeda A, Park M et al. Hypoxia promotes expansion of the CD133positive glioma stem cells through activation of HIF-1alpha. Oncogene 2009; 28 (45): 3949-3959.

30. Yao J, Zhang T et al. Effect of CD133/prominin-1 antisense oligodeoxynucleotide on in vitro growth characteristics of Huh-7 human hepatocarcinoma cells and U251 human glioma cells. Oncol Rep 2009; 22 (4): 781-787.

Received February 12, 2014. Accepted March 8, 2015. 